\title{
DILATAÇÃO DOS ESPAÇOS DE VIRCHOW-ROBIN EM PACIENTES COM MIGRÂNEA
}

\author{
Marcos Alberto da Costa Machado Júnior', Adriana Silva Matos², \\ Fabíola Goyanna², Verônica Aline Oliveira Barbosa' ${ }^{1}$ Lauro Conceição Vieira ${ }^{3}$
}

\begin{abstract}
RESUMO - Os espaços de Virchow-Robin (EVR) são invaginações do espaço subpial, formando uma bainha de revestimento tubular contendo um vaso, que separa o espaço subaracnóide do subpial. A ressonância magnética (RM) é o único método de imagem capaz de avaliar este detalhe anatômico. Nós estudamos a possível associação entre EVR dilatados e migrânea. Avaliamos 70 pacientes com idade compreendida entre 13 e 54 anos (média 36,5 anos), com diagnóstico clínico de migrânea e comparamos com grupo controle com igual número de pacientes com idade compreendida entre 14 e 64 anos (média de 42 anos), sem antecedentes de cefaléia. Observamos aumento dos EVR em 28 casos (40\%) dos pacientes com migrânea. No grupo controle tal achado foi encontrado em apenas 5 casos (7,1\%). Alertamos, ainda, sobre a importância na detecção e reconhecimento dos EVR, bem como o seu diagnóstico diferencial com infartos lacunares e cistos da fissura coroidea.
\end{abstract}

PALAVRAS-CHAVE: espaços perivasculares de Virchow-Robin, ressonância magnética, migrânea.

\begin{abstract}
Dilatation of Virchow-Robin spaces in patients with migraine
ABSTRACT - The Virchow-Robin spaces (VRS) are subpial invaginations making a tubular revestment scabbard that contains a vase between the subarachnoid and subpial spaces. The magnetic resonance imaging (MRI) is the only imaging method able for evaluate this anatomic detail. We studied the possible association between dilated VRS and migraine. Seventy patients with ages ranging from 13 to 54 years (mean, 36.5 years), with clinical diagnosis of migraine were studied and compared with a control group, without past of headache, composed by the same number of patients with ages ranged from 14 to 64 years (mean, 42 years). We observed widening of VRS in 28 cases $(40 \%)$ of the migraine patients. In the control group this feature was demonstrated in only 5 cases $(7.1 \%)$. Alert for the importance of detecting and recognizing the VRS, as well as, the diferentiation from lacunar infarctions and coroid fissure cists.
\end{abstract}

KEY WORDS: migraine, Virchow-Robin spaces, magnetic resonace imaging.

No passado, acreditava-se que os espaços de Virchow-Robin (EVR) eram invaginações dos espaços subaracnóides ${ }^{1,2}$. Estudos com microscopia eletrôni$\mathrm{ca}$, sugerem que os EVR originam-se de invaginações do espaço subpial, formando uma bainha de revestimento que contém as artérias separando o espaço subaracnóide do subpial ${ }^{3,4}$.

A dilatação dos EVR é facilmente detectada pela ressonância magnética $(\mathrm{RM})^{5}$ e tem sido descrita em pacientes com criptococose ${ }^{6}$, mucopolissacaridose ${ }^{7}$, crianças com cefaléia ${ }^{8}$, envelhecimento ${ }^{9}$ macrocefalia e epilepsia do lobo temporal ${ }^{10}$ ou ainda, em achados ocasionais concebidos como variantes do normal ${ }^{11,12}$.

Nós revimos 70 RM de pacientes com migrânea e comparamos com igual número de pacientes sem antecedentes de cefaléia.

\section{MÉTODO}

Setenta exames de RM de pacientes com Migrânea foram realizados no período compreendido entre 1/Janeiro/1999 e 7/Julho/2000, em pacientes com idade compreendida entre 13 e 54 anos (média 36,5 anos), com diagnóstico clínico de migrânea e sem outra queixa neurológica. Comparamos com igual número de controle com idade compreendida entre 14 e 64 anos (média 42 anos), sem antecedentes de cefaléia, submetidos ao estudo RM do crânio para estadiamento de lesões neoplásicas secundárias, sem acometimento do SNC e quadro clínico de migrânea.

Usamos aparelhagem de RM de 1,5T (GE.- Signa Horison LX.) e com as seguintes sequências:

FSE - Imagem ponderada em T2 com cortes axiais e coronais (TR 4.500, TE 90), com $5 \mathrm{~mm}$ de espessura.

Imagem ponderada em DP com cortes axiais e coronais (TR 200, TE 35), $5 \mathrm{~mm}$ de espessura.

Trabalho realizado no Setor de Neurorradiologia do Serviço de Bioimagem do Hospital São Rafael - Fundação Monte Tabor, Salvador Bahia: ${ }^{1}$ Neurorradiologista; ${ }^{2}$ Residentes em Radiologia; ${ }^{3}$ Técnico do Serviço de Bioimagem.

Recebido 15 Setembro 2000, recebido na forma final 8 Dezembro 2000. Aceito 12 Dezembro 2000. 

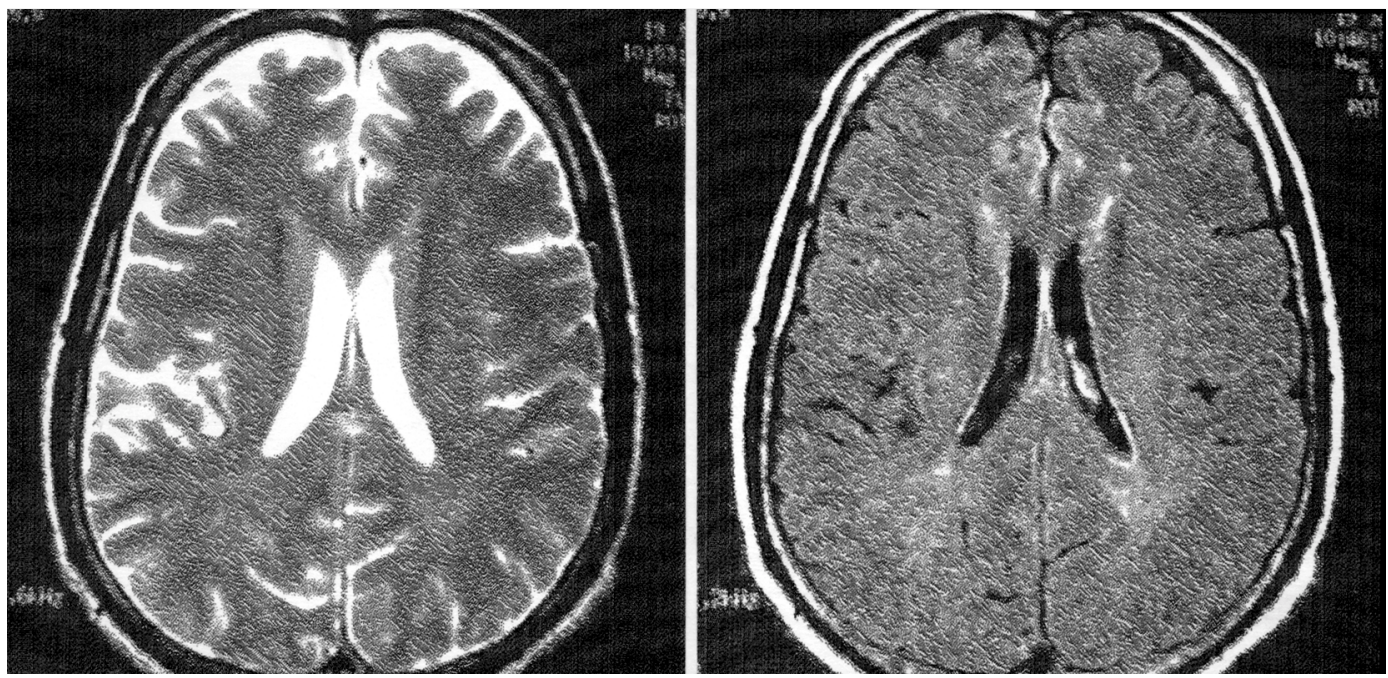

Fig 1. A - Axial T2. B - Imagem FLAIR. EVR dilatados em paciente idoso. Veja a orientação no sentido das artérias penetrantes corticais. Assinala-se área de hipersinal na imagem FLAIR traduzindo perda de mielina em situação periventricular frontal esquerda de provável natureza vascular.

SE - Imagem ponderada em T1 com cortes axiais e sagitais. (TR 500, TE 14), com $5 \mathrm{~mm}$ de espessura.

FLAIR - Fluid Atenuation Invertion Recovery (Inversão Recuperação com Supressão de água livre).

- Cortes axiais (TR 10.002, TE 170, TI 2.200), com 5 mm de espessura.

Utilizamos seqüência SE com imagem ponderada em T1 com cortes axiais e sagitrais. (TR 500, TE 14), com 5 $\mathrm{mm}$ de espessura após a administração venosa do contraste paramagnético - Gadolínio - em todos os pacientes, inclusive, no grupo controle.

A matriz utilizada foi 256 X 256. Os EVR foram caracterizados como: imagens redondas ou ovais, com margens lisas e bem definidas, sem efeito compressivo, sem realce das paredes após gadolínio, de dimensões superiores a $0,5 \mathrm{~cm}$ e com identificação de arteríolas no interior. Localização típica: adjacente à comissura anterior, porção basal dos núcleos cinza, coroa radiada, mesencéfalo, entre a substância nigra e a divisão dos pedúnculos cerebelares superiores. Preferencialmente no trajeto das arteríolas perfurantes corticais, perfurantes mesencefálicas e lenticuloestriadas. Os exames foram avaliados por dois neurorradiologistas, independentes, e as discordâncias de interpretação dirimidas posteriormente.

Foram considerados aumentos patológicos dos EVR, quando vistos na substância branca da coroa radiada, uni ou bilateralmente, com intensidade de sinal idêntica à da água livre em todas as seqüências. Não foram considerados aumentados, quando nas imagens em DP e FLAIR não eram vistos, pois, com dimensões reduzidas aparecem com intensidade de sinal igual a do parênquima adjacente.

\section{RESULTADOS}

O aumento dos EVR esteve presente em 28 casos (40\%) dos pacientes com migrânea. No grupo con- trole tal achado foi encontrado em apenas 5 casos $(7,1 \%)$.

As localizações mais freqüentes foram nos centros semi-ovais, adjacentes à comissura anterior, mesencéfalo e pedúnculos cerebelares.

Áreas focais de hiperintensidade de sinal em T2 e seqüência FLAIR foram observadas em ambos os grupos, em $60 \%$ dos pacientes acima dos 55 anos, com localização supratentorial, acometendo a substância branca da coroa radiada, bilateralmente, traduzindo perda de mielina. Em 15\% destes foram observadas lesões hipointensas em $\mathrm{T} 1$ e hiperintensa em T2 e FLAIR, traduzindo gliose devido a infartos lacunares nos núcleos da base e coroa radiada.

\section{DISCUSSÃO}

A dilatação dos EVR tem sido relatada em associação com o envelhecimento fisiológico, hipertensão arterial e demência ${ }^{13}$. Há uma nítida associação estatística entre a idade e o alargamento dos EVR, sugerindo que seja mais um fenômeno associado ao envelhecimento encefálico, similar ao que ocorre com os espaços subaracnóides (Fig 1). Recentemente, alguns autores têm associado a cefaléia ao aumento dos EVR. Schick e cols., evidenciaram aumento dos EVR em $61 \%$ das crianças com migrânea e $22 \%$ com cefaléia tensional em relação ao grupo controle ${ }^{14}$.

A dilatação dos EVR é facilmente detectada pela $\mathrm{RM}^{5}$ sendo descrita em pacientes com criptococose ${ }^{6}$, mucopolissacaridose com acúmulo intracelular, nos EVR de glicosaminoglicanas ${ }^{7}$, em trabalhos estatísticos com crianças com cefaléia ${ }^{8}$, envelhecimento sem alterações neurológicas, notadamente, no he- 

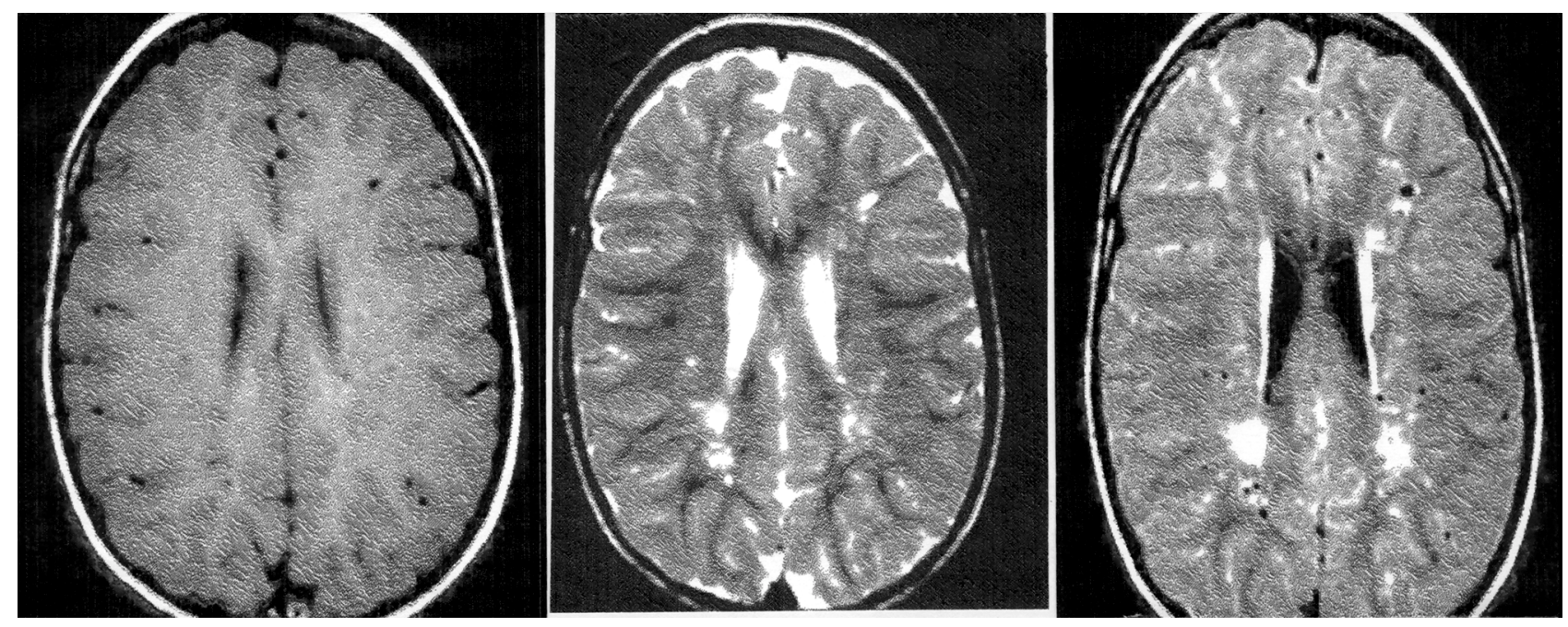

Fig 2. A - Axial T2. B - Axial T2. C - Imagem FLAIR. Acentuado grau de perda de mielina peritrigonal e EVR amplos possivelmente existem diminutas áreas de porencefalia associadas.

misfério cerebral esquerdo ${ }^{9}$, macrocefalia e epilepsia do lobo temporal associado ao aumento do sulco hipocampal ${ }^{10}$. Ou, ainda, em achados ocasionais em pacientes sem anormalidades fisiológicas ${ }^{11,12}$, presumivelmente variantes do normal. Schick e cols., consideram variantes anatômicas os EVR amplos quando adjacentes à comissura anterior e tronco encefálico ${ }^{14}$.

Rollins e cols. ${ }^{8}$, realizaram estudo evolutivo que não evidenciou progressão das dimensões dos EVR após 1 ano, em crianças com cefaléia crônica ou recorrente. Estudos futuros, serão necessários para elucidar se a migrânea pode promover ou determinar alterações estruturais, e se, tais achados, são manifestações prematuras de malformações neurotransmissoras $^{15}$.

Existem relatos, também, da elevada freqüência de microinfartos e gliose focal em migranosos em comparação com grupo controle sem migrânea. Correlacionando lesões focais da substância branca e a produção de microembolos e infartos lacunares secundários ao aumento da agregação plaquetária e liberação de agentes vasoconstrictores, como a serotonina ${ }^{16,17}$. No nosso trabalho, não evidenciamos prevalência de alterações demielinizantes e nem de lesões glióticas de etiologia vascular entre os grupos.

Várias hipóteses têm sido sugeridas para o mecanismo da patogênese do aumento das dimensões dos EVR, como o efeito das pulsações liquóricas, fenômenos atróficos, ectasia vascular, permeabilidade anormal da parede vascular ${ }^{18}$.

Esiri sugere que os EVR, sejam tecido conectivo modificado, contendo líquido cefalorraquidiano (LCR) ao invés de linfa, e que funcionando como um compartimento imunológico encefálico hipertrofiado, tornando-se repleto de células inflamatórias sob estimulação imunológica do sistema nervoso central (SNC). Sabe-se, que em cobaias, quando os linfáticos cervicais são obstruídos os EVR distendemse devido ao acúmulo de líquido proteináceo, ocorrendo também edema cerebral e papiledema. É conhecida, a associação dos linfáticos das paredes das artérias cerebrais do polígono de Willis com os EVR. Ocorre também comunicação entre o espaço subaracnóide (ESA) e linfáticos ao longo dos nervos cranianos e espinhais ${ }^{18}$.

O significado de uma via de drenagem do LCR em direção ao sistema linfático é que, antígenos presentes no LCR, espaço extracelular ou tecido encefálico, podem ser captados por macrófagos perivasculares e transportados para linfáticos cervicais para serem reconhecidos por linfócitos T e linfócitos $B^{19}$. Assim sendo, os EVR parecem ter importante atuação nas reações imunológicas das enfermidades infecciosas e neoplásicas. Esta última, disseminação de glioma maligno, tem sido documentada através da parede dos vasos para o espaço subpial (ESP) e para o ESA. Na leucemia e no linfoma as células tumorais migram para o parênquima profundo através dos EVR. Alguns carcinomas permanecem limitados ao ESA, com invasão microscópica do encéfalo, enquanto outros penetram no ESP, disseminando-se profundamente pelo parênquima através dos EVR ou por invasão da glia limitans².

O principal diagnóstico diferencial dos EVR proeminentes, deve ser feito com lesões isquêmicas lacunares, nos territórios das artérias lentículoestriadas, perfurantes mesencefálicas e corticais. Os infartos 

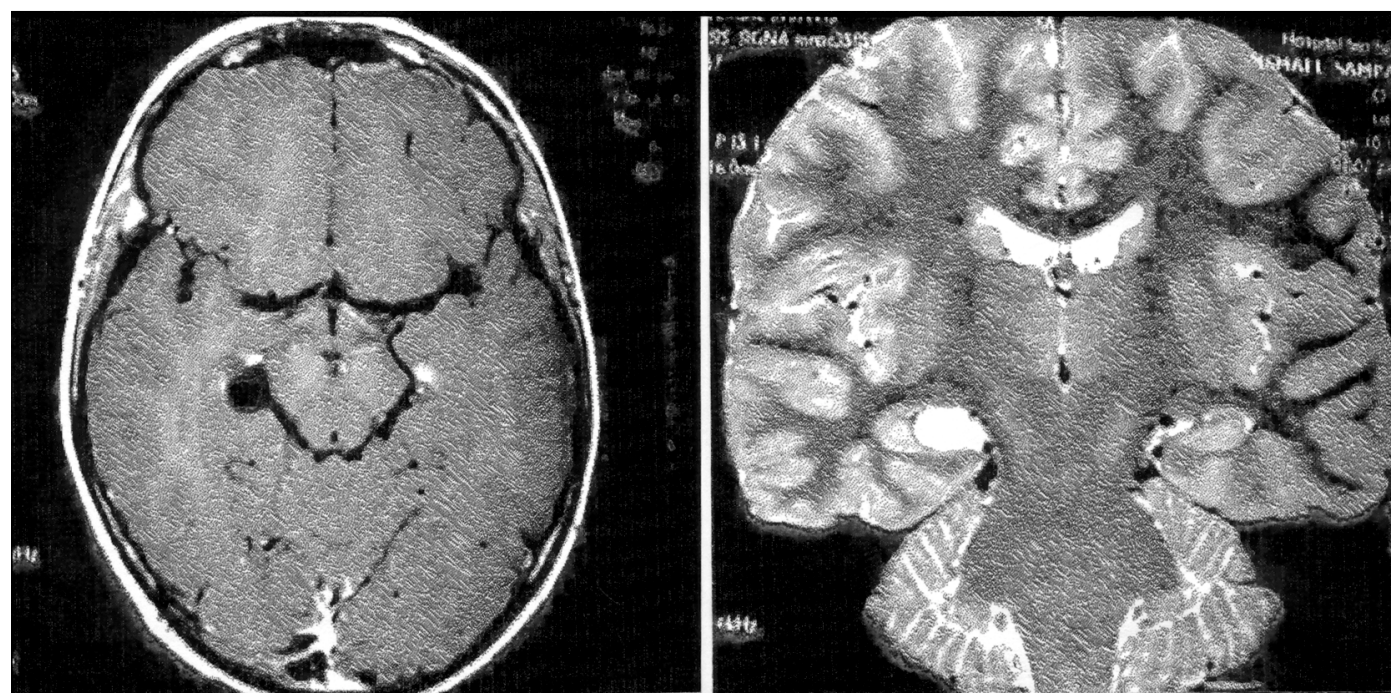

Fig 3. Cisto da fissura coroidea. A - T1 axial após gadolínio. Vê-se leve efeito compressivo sobre o corno inferior adjacente. $\boldsymbol{B}$ - Coronal T2 Imagem cística insinuando-se pela fissura coroidea. Não se observam estruturas vasculares no seu interior.

possuem sinal elevado nas seqüências FLAIR e nas imagens em densidade de prótons e acometem mais os terços superiores dos núcleos da base (Fig 1). Os espaços de VR são isointensos em relação ao líquor, em todas as seqüências, com configuração centrípeta acompanhando a direção das arteríolas penetrantes. As lesões isquêmicas, quando em fase aguda ou subaguda, são facilmente diferenciadas com a técnica de difusão.

O diagnóstico diferencial deve ser feito também, com diminutas cavidades porencefálicas, de etiologia anóxico-isquêmica perinatal associadas à fenômenos atróficos, podendo ambos achados coexistirem (Fig 2). Outro diagnóstico diferencial é com cistos da fissura coroidea. Nestes, não se observam vasos no interior (Fig 3).

O diagnóstico diferencial com cérebro policístico (imagens "císticas" associadas à displasia ectodérmica) pode na realidade corresponder a EVR dilatados.

\section{REFERÊNCIAS}

1. Maynard EA; Schultz RL; Pease DC. Electron microscopy of the vascular bed of rat cerebral cortex. Am J Anat 1958;102:301-321.

2. Millen JW, Woollan DHM. On the nature of the pia mater. Brain 1961;84:514-520.

3. Zhang ET, Inman CBE, Weller RO. Interrelationships of the pia mater and the perivascular (Virchow-Robin) spaces in the human cerebrum. J Anat 1990;170:11-123.
4. Nicholas DS, Weller RO. The fine anatomy of the human spinal meninges. A light and scanning electron microscopy study. J Neurosurg 1988;69:276-282.

5. Weller RO. Anatomy and pathology of the subpial space. Riv Neuroradiol 1994;7(Supp 14):15-21.

6. Kumazada K, Yamada T. Serial MRI findings in patients with CNS cryptococcosis. Rinsho Shinkeigaku 1998;38:831-837.

7. Murata R, Nakajima S, Tanaka A, Miyagi N, Matzuoka 0, Kogame S, Inoue Y. MR imaging of the brain in patients with mucopolysaccharidosis. AJNR 1989;10:1165-1170.

8. Rollins NK, Deline C, Morriss MC. Prevalence and clinical significance of dilated Virchow-Robin spaces in childhood. Radiology 1993;189:53-57.

9. Chuang YC, Lui CC.Unsual dilatation of Virchw-Robin spaces: case report. Chang Keng I Hsueh Tsa Chih 1999;22:671-675.

10. Bastos AC, Andermann F. Late-onset temporal lobe epilepsy and dilatation of the hippocampal sulcus by an enlarged Virchow-Robin space. Neurology 1998;50:784-787.

11. Ugawa Y, Shirouzu I, Terao Y. Physiological analyses of patient with extreme widening of Virchow-Robin spaces. J Neurol Sci 1998;159:25-27.

12. Adachi M, Hosoya T, Haku T. Dilated Virchow-Robin spaces: MRI pathological study. Neuroradilogy 1998;40:27-31.

13. Heier LA, Bauer CJ, Schwartz L, Zimmerman RD, Morgello S, Deck MD. Large Virchow-Robin spaces: MR-clinical correlation. AJNR 1999;10:929-936.

14. SchickS, Gahleitner, Wober-Bingol. Virchow-Robin spaces in childhood migraine. Neuroradiology 1999;41:283-287.

15. Enierit MB, Riad M, Hamon M. Trophic effects of neurotransmitters during brain maturation. Biol Neonate 1992;62:193-201

16. De Benedittis B, Lorenzetti A, Siana C, Bernasconi V. Magnetic resonance imaging in migraine and tension-type headache. Headache 1995;35:264-268.

17. Fazekas F, Koch M, Schmidt R, Often- bacher H, Payer F, Freidi W, Lechner $H$. The prevalence of cerebral damage varies with migraine type: a MRI study. Headache 1992;32:287-291.

18. Mirfakhraee M, Crofford MJ, Guinto FC. Virchow-Robin space: a.path of spread in neurosarcoidosis. Radiology 1986;158:715-720.

19. Esiri MM, Gay D. Immunological and neuropathological significance of the Virchow-Robin spaces. J Neurol Sci 1990;100:3-8. 